Conclusion JB01-BD is safe and effective against HPV and HIV infections, and was approved by China Food and Drug Administration (CFDA) for treating cervical HPV infection with the potential to reduce morbidity of cervical cancer. A lubricant formulation containing JB01 has recently been approved by CFDA as an auxiliary strategy to prevent sexual transmission of HIV.

Disclosure No significant relationships.

\section{P840 THE HPV SCREENING AND VACCINE EVALUATION (HPV- SAVE) STUDY IN MEN LIVING WITH HIV: EARLY PATHOLOGIC AND ACCEPTABILITY OUTCOMES}

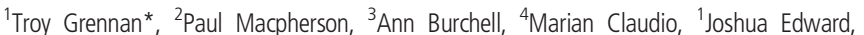
${ }^{3}$ Jennifer Gillis, ${ }^{5}$ Daniel Grace, ${ }^{6}$ Ronita Nath, ${ }^{4}$ Ron Rosenes, ${ }^{7}$ Darrell Tan, ${ }^{4}$ Irving Salit. ${ }^{1} B C$ Centre for Disease Control, Clinical Prevention Services, Vancouver, Canada; ${ }^{2}$ The Ottawa Hospital, Ottawa, Canada; ${ }^{3}$ St. Michael's Hospital, Li Ka Shing Knowledge Institute, Toronto, Canada; ${ }^{4}$ University Health Network, Toronto, Canada; ${ }^{5}$ University of Toronto, Dalla Lana School of Public Health, Toronto, Canada; ${ }^{6}$ British Columbia Centre for Disease Control, Clinical Prevention, Vancouver, Canada; ' 5 t. Michael's Hospital, Toronto, Canada
\end{abstract}

\subsection{6/sextrans-2019-sti.885}

Background Anal cancer caused by oncogenic, high-risk (HR) human papillomavirus (HPV) is emerging as a leading cause of non-HIV-related death in HIV-positive MSM. Anal cancer rates in HIV-positive MSM are up to 100-times higher than the general population. There are no universally-accepted guidelines for anal cancer screening, even in high risk populations, due to a paucity of evidence to support its effectiveness. We assessed the acceptance rate to invitations for anal cancer screening, and describe preliminary pathology results.

Methods The HPV-SAVE Study is an ongoing Canadian study on screening and treatment of anal cancers and pre-cancers in HIV-positive MSM. Participants were invited to have anal cytology and HPV testing in their physician's office. Those with abnormalities were referred for high resolution anoscopy (HRA) and anal biopsies. Cytology was graded as per the Bethesda classification, and histology was described per the Lower Anogenital Squamous Terminology (LAST) nomenclature.

Results Out of 2241 invitations as of 01/2019, 617 men $(27.5 \%)$ agreed to be screened. Cytology results from 518 satisfactory Pap tests were: 246 negative (47.5\%), 62 LSIL (12.0\%), 9 LSIL-H (1.7\%), 14 HSIL (2.7\%), 174 ASCUS (33.6\%), and 13 ASC-H (2.5\%). In 116 participants referred for HRA, 247 biopsies were done, yielding HSIL in 62 $(53.4 \%)$ unique individuals, and one invasive carcinoma. In a sample of 127 participants, $111(87.4 \%)$ had any HPV type, $82(64.6 \%)$ had multiple HPV types, 78 (61.4\%) had at least one high-risk HPV type, and 39 (30.7\%) had HPV-16.

Conclusion MSM living with HIV had moderate acceptance of anal cancer screening invitations, with over half of screened men having abnormal cytology. A majority of those undergoing HRA had high-grade histology diagnosed, and most participants had HPV anal canal infection, with nearly two-thirds having anal canal infection with HR-HPV. These early results highlight the enormous HPV burden in this high-risk population.

Disclosure No significant relationships.

\section{P841 BARRIERS TO HPV VACCINATION AMONG GAY, BISEXUAL, AND OTHER MEN WHO HAVE SEX WITH MEN (GBMSM) IN CANADA: A CIRN STUDY}

${ }^{1}$ Ramandip Grewal ${ }^{*},{ }^{1}$ Anna Yeung, ${ }^{2}$ Marc Brisson, ${ }^{3}$ Troy Grennan, ${ }^{4}$ Alexandra De Pokomandy, ${ }^{4}$ Joseph Cox, ${ }^{5}$ Gilles Lambert, ${ }^{6}$ David Moore, ${ }^{7}$ François Coutlée, ${ }^{8}$ Shelley Deeks, ${ }^{9}$ Sandra Gardner, ${ }^{10}$ Dane Griffiths, ${ }^{11}$ Wanrudee Isaranuwatchai, ${ }^{12}$ Jody Jollimore, ${ }^{13}$ James Murray, ${ }^{14}$ Gina Ogilvie, ${ }^{15}$ Chantal Sauvageau, ${ }^{1}$ Darrell Tan, ${ }^{16}$ Barry Adam, ${ }^{14}$ Heather Armstrong, ${ }^{9}$ Mark Gaspar, ${ }^{17}$ Clemon George, ${ }^{18}$ Daniel Grace, ${ }^{19}$ Trevor Hart, ${ }^{1}$ Ann Burchell. 'St. Michael's Hospital, Centre For Urban Health Solutions, Li Ka Shing Knowledge Institute, Toronto, Canada; ' Université Laval, Quebec City, Canada; ${ }^{3} B C$ Centre for Disease Control, Clinical Prevention Services, Vancouver, Canada; ${ }^{4}$ McGill University, Montreal, Canada; ${ }^{5}$ University of Montreal, Montreal, Canada; ${ }^{6} B C$ Centre for Excellence in HIVIAIDS, Vancouver, Canada; 'I'Université de Montréal, Microbiologie Médicale et Infectiologie, Montreal, Canada; ${ }^{8}$ Public Health Ontario, Toronto, Canada; ${ }^{9}$ University of Toronto, Toronto, Canada; ${ }^{10}$ Gay Men's Sexual Health Alliance, Toronto, Canada; ${ }^{11}$ St. Michael's Hospital, Toronto, Canada; ${ }^{12}$ Community Based Research Centre, Vancouver, Canada; ${ }^{13}$ Ontario Ministry of Health and Long-Term Care, Toronto, Canada; ${ }^{14}$ University of British Columbia, Vancouver, Canada; ${ }^{15}$ Institut National de Santé Publique du Québec, Montreal, Canada; ${ }^{16}$ University of Windsor, Windsor, Canada; ${ }^{17}$ University of West Indies - Cave Hill, Cave Hill, Barbados; ${ }^{18}$ University of Toronto, Dalla Lana School of Public Health, Toronto, Canada; ${ }^{19}$ Ryerson University, Psychology, Toronto, Canada

\subsection{6/sextrans-2019-sti.886}

Background Internationally, Canada was one of the first countries to offer free HPV vaccination to young gbMSM. The provinces of British Columbia (BC), Ontario (ON) and Quebec (QC) implemented HPV vaccination programs in 20152016 for gbMSM aged 9-26. We explored HPV vaccine initiation and among unvaccinated men, potential barriers to uptake.

Methods Engage is a sexual health study among gbMSM aged $16+$ in Vancouver BC, Toronto ON, and Montreal QC, the largest urban centres in each province. Men are recruited via respondent driven sampling (RDS). We estimated the proportion that had initiated HPV vaccination and among unvaccinated men, compared proportions to responses on HPV vaccine knowledge and willingness, healthcare access and sexual orientation disclosure by age (eligible for free vaccination: $\leq 26$ and ineligible for free vaccination: $\geq 26$ years old). Proportions were RDS unadjusted.

Results From 01/2017 to 31/12/2018, 2099 men enrolled (542 Vancouver, 378 Toronto, 1179 Montreal). Their median age was 33 years (IQR 27-46). In Vancouver, Toronto, and Montreal, $48.6 \%, 44.1 \%$ and $44.2 \%$ of men aged $\leq 26$ and $24.9 \%$, $30.3 \%$ and $7.1 \%$ of men aged $\geq 26$ had $1+$ dose of the HPV vaccine, respectively. Among unvaccinated men, more men aged $\geq 26$ versus $\leq 26$ accessed healthcare (family doctor/sexual health/HIV care) $(87.2 \%$ vs $78.9 \%, \mathrm{p}=0.0008)$ and disclosed their sexual orientation to their family doctor $(86.2 \%$ vs $64.4 \%, \mathrm{p}<0.0001)$. A similar proportion of unvaccinated men from each age group had heard of the HPV vaccine $(\geq 26=67.5 \%$ vs $\leq 26=69.0 \%, p<0.0529)$. Among unvaccinated men aware of the HPV vaccine, more men aged $\geq 26$ versus $\leq 26$ were willingness to get vaccinated if the vaccine were free and required disclosure of same-sex activity $(80.3 \%$ vs $61.5 \%, \mathrm{p}=0.0259)$.

Conclusion Preliminary analyses indicate that HPV vaccine initiation in this population remains suboptimal. Among unvaccinated gbMSM $\leq 26$, accessing healthcare, not disclosing sexual orientation and willingness to get vaccinated may be barriers to vaccine uptake.

Disclosure No significant relationships. 